expression was highly variable and distinct PD-L1 driven immune phenotypes were identified based on the PD-L1 intensity on both tumor and immune cells, the distance between non-exhausted T-cell subsets (i.e. PD-1 and CTLA-4 expression on $\mathrm{CD}^{+}{ }^{+} \mathrm{CD} 8{ }^{+}$cytotoxic $\mathrm{T}$-cells, $\mathrm{CD} 3{ }^{+} \mathrm{CD} 4^{+} \mathrm{T}-$ helper cells, $\mathrm{CD}^{+}{ }^{+} \mathrm{CD} 4{ }^{+} \mathrm{FOXP} 3^{+}$regulatory $\mathrm{T}$-cells) and tumor cells as well as macrophage/(DC) subtypes. In breast cancer, the PD-L1 fluorescence intensity on tumor cells showed a significantly higher predictive performance for overall survival with an area under receiver operating curves (AUC) of $0.72(\mathrm{p}<0.0001)$ than the percentage of PD-L1 ${ }^{+}$ tumor cells (AUC: 0.54). In PD-L1 positive as well as negative breast cancers a close spatial relationship between $\mathrm{T}$ - cell subsets $\left(\mathrm{CD} 3{ }^{+} \mathrm{CD} 4{ }^{ \pm} \mathrm{CD} 8{ }^{ \pm} \mathrm{FOXP} 3{ }^{ \pm} \mathrm{PD}-1^{ \pm} \mathrm{CTLA}-4^{ \pm}\right)$and Macrophage/DC subsets $\left(\mathrm{CD} 68^{ \pm} \mathrm{CD} 163^{ \pm} \mathrm{CD} 11 \mathrm{c}^{\left.{ }^{ \pm} \mathrm{iNOS}\right)}\right.$ was found prognostic relevant $(p<0.0001)$.

Conclusions In conclusion, multiplex immunofluorescence PD-L1 assessment provides cutoff-free/continuous PD-L1 data which are superior to the conventional percentage of $\mathrm{PD}-\mathrm{L} 1^{+}$tumor cells and of high prognostic relevance. The combined analysis of spatial PD-L1/PD-1 data and more than 20 different immune cell subtypes of the immune tumor microenvironment revealed distinct PD-L1 immune phenotypes.

Disclosure Information N.C. Blessin: None. E. Bady: None. T. Mandelkow: None. C. Yang: None. J. Raedler: None. R. Simon: None. C. Fraune: None. M. Lennartz: None. S. Minner: None. E. Burandt: None. D. Höflmayer: None. G. Sauter: None. S.A. Weidemann: None.

\section{P02.04 TISSUE-INFILTRATING TH9 CELLS IN HUMAN ENDOMETRIAL CANCER}

${ }^{1} \mathrm{H}$ Tong ${ }^{*}, 2,3,4 \mathrm{H}$ Feng, ${ }^{1} \mathrm{X}$ Wan. ${ }^{1}$ Shanghai First Maternity and Infant Hospital, Shanghai, China; ${ }^{2}$ Department of Liver Surgery, Renji Hospital, School of Medicine, Shanghai Jiao Tong University, Shanghai, China; ${ }^{3}$ Shanghai Institute of Transplantation, Shanghai, China; ${ }^{4}$ Alumni Network of Ludwig-Maximilians University of Munich, Shanghai, China

\subsection{6/jitc-2021-ITOC8.16}

Background Endometrial cancer (EC) is a hormone-related carcinoma with increased morbidity among female patients of all backgrounds. The immune microenvironment of EC is uncertain.

Materials and Methods 102 patients were recruited in the present study. 90 postoperative specimens from the patients were analyzed by immunohistochemistry. The leukocyte landscape of endometrial cancer was mapped using high-dimensional single-cell profiling (CyTOF) for 12 patients.

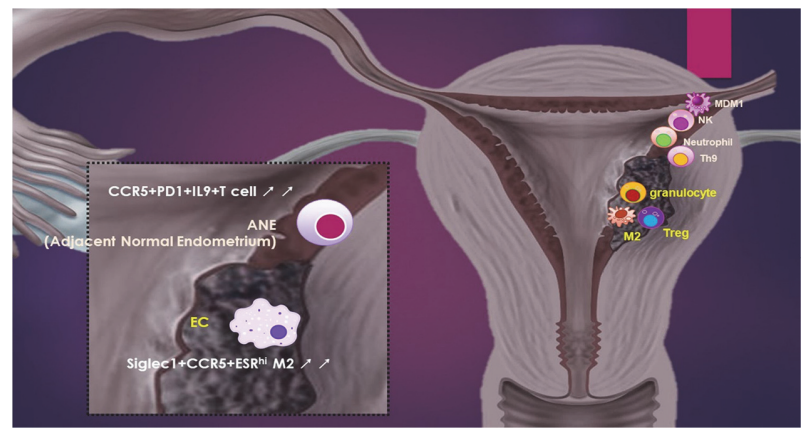

Abstract P02.04 Figure 1
Results NK cells, MDMs, and neutrophils were enriched in adjacent normal tissue. CCR5 + CD38+ PD1+Th9 cells were enriched in the invasive margin. Additionally, PD1+ESR $\mathrm{Eng}_{\text {eg }} \mathrm{T}$ cells and Siglec1+CCR5 +CD40+ESR ${ }^{\text {hi }}$ macrophage were infiltrated in the tumors.

Conclusions Immunological landscape of EC might shed light on new immunotherapuetic approach.

Disclosure Information H. Tong: None. H. Feng: None. X. Wan: None.

\section{P02.05 COMPREHENSIVE PROFILING OF TUMOR HETEROGENEITY AND ITS MICROENVIRONMENT IN ADVANCED NON-SMALL CELL LUNG CANCER AT SINGLE CELL RESOLUTION}

${ }^{1} \mathrm{Y}$ Cordts*, ${ }^{2} \mathrm{~F}$ Wu, ${ }^{1} \mathrm{~J}$ Fan, ${ }^{3} \mathrm{M}$ Odenthal, ${ }^{3} \mathrm{R}$ Buettner, ${ }^{2} \mathrm{C}$ Zhou. ${ }^{1}$ Singleron Biotechnologies, Cologne, Germany; ${ }^{2}$ Shanghai Pulmonary Hospital, Shanghai, China; ${ }^{3}$ University Hospital Cologne, Cologne, Germany

\subsection{6/jitc-2021-ITOC8.17}

Background Lung cancer is a highly heterogeneous disease. Cancer cells and cells within the tumor microenvironment together determine disease progression, as well as response to or escape from treatment.

Materials and Methods To map the cell type-specific transcriptome landscape of cancer cells and their tumor microenvironment in advanced non-small cell lung cancer (NSCLC), we analyzed 42 tissue biopsy samples from stage III/IV NSCLC patients by single cell RNA sequencing and presented the large scale, single cell resolution profiles of advanced NSCLCs.

Results In addition to cell types described in previous single cell studies of early stage lung cancer, we were able to identify new cell types such as follicular dendritic cells and $T$ helper 17 cells. Tumors from different patients display large heterogeneity in cellular composition, chromosomal structure, developmental trajectory, intercellular signaling network and phenotype dominance. Our study also revealed a correlation of tumor heterogeneity with tumor associated neutrophils, which might help to shed light on their function in NSCLC. Conclusions This study presented first-time the tumor heterogeneity and tumor microenvironment profile from late-stage, largely untreated NSCLC patients, and shed light on possible treatment regimes.

Disclosure Information Y. Cordts: A. Employment (full or part-time); Modest; Singleron Biotechnologies GmbH. F. Wu: None. J. Fan: A. Employment (full or part-time); Modest; Singleron Biotechnologies GmbH. M. Odenthal: None. R. Buettner: None. C. Zhou: None.

\section{P02.06 SEMI-AUTOMATED VALIDATION AND QUANTIFICATION OF CTLA-4 IN 90 DIFFERENT TUMOR ENTITIES USING MULTIPLE ANTIBODIES AND ARTIFICIAL INTELLIGENCE}

D Dum, TLC Henke, T Mandelkow, E Bady, R Simon, G Sauter, S Steuerer, W Wilczak, E Burandt, J Raedler, M Lennartz, NC Blessin*. University Medical Center HamburgEppendorf, Hamburg, Germany

\subsection{6/jitc-2021-ITOC8.18}

Background CTLA-4 is an inhibitory immune checkpoint receptor and a negative regulator of anti-tumor T-cell 
function. This study aimed at a comparative analysis of CTLA- $4^{+}$cells between different tumor entities.

Materials and Methods To quantify CTLA $-4^{+}$cells, 4,582 tumor samples from 90 different tumor entities as well as 608 samples of 76 different normal tissue types were analyzed by immunohistochemistry in a tissue microarray format. Two different antibody clones (MSVA-152R and CAL49) were validated and quantified using a deep learning framework for automated exclusion of unspecific immunostaining.

Results Comparing both CTLA-4 antibodies revealed a clone dependent unspecific staining pattern in adrenal cortical adenoma (63\%) for MSVA-152R and in pheochromocytoma $(67 \%)$ as well as hepatocellular carcinoma (36\%) for CAL49. After automated exclusion of non-specific staining reaction (3.6\%), a strong correlation was observed for the densities of CTLA $-4^{+}$lymphocytes obtained by both antibodies $(r=0.87$; $\mathrm{p}<0.0001)$. The mean density of $\mathrm{CTLA}^{-} 4^{+}$cells was 674 \pm 1482 cells $/ \mathrm{mm}^{2}$ and ranged from $71 \pm 175$ cells $/ \mathrm{mm}^{2}$ in leiomyoma to $5916 \pm 3826$ cells $/ \mathrm{mm}^{2}$ in Hodgkin's lymphoma. Within epithelial tumors, the density of CTLA-4 ${ }^{+}$ lymphocytes were higher in squamous cell (421 \pm 467 cells/ $\left.\mathrm{mm}^{2}\right)$ and urothelial carcinomas $\left(419 \pm 347\right.$ cells $\left./ \mathrm{mm}^{2}\right)$ than in adenocarcinomas $\left(269 \pm 375\right.$ cells $\left./ \mathrm{mm}^{2}\right)$ and renal cell neoplasms $\left(256 \pm 269\right.$ cells $\left./ \mathrm{mm}^{2}\right)$. A high CTLA $-4^{+}$cell density was linked to low pT category $(\mathrm{p}<0.0001)$, absent lymph node metastases $(p=0.0354)$, and PD-L1 expression in tumor cells or inflammatory cells $(\mathrm{p}<0.0001$ each). A high CTLA-4/ CD3-ratio was linked to absent lymph node metastases $(\mathrm{p}=0.0295)$ and to PD-L1 positivity on immune cells $(\mathrm{p}<0.0026)$.

Conclusions Marked differences exist in the number of CTLA$4+$ lymphocytes between tumors. Analyzing two independent antibodies by a deep learning framework can facilitate automated quantification of immunohistochemically analyzed target proteins such as CTLA-4.

Disclosure Information D. Dum: None. T.L.C. Henke: None. T. Mandelkow: None. E. Bady: None. R. Simon: None. G. Sauter: None. S. Steuerer: None. W. Wilczak: None. E. Burandt: None. J. Raedler: None. M. Lennartz: None. N.C. Blessin: None.

\section{P02.07 CHARACTERIZATION OF THE TUMOR IMMUNE MICROENVIRONMENT OF PEDIATRIC POSTERIOR FOSSA A EPENDYMOMAS}

${ }^{1} \mathrm{~J}$ Lammers*, ${ }^{1} \mathrm{~F}$ Calkoen, ${ }^{1} \mathrm{M}$ Kranendonk, ${ }^{2,3} \mathrm{~A}$ Federico, ${ }^{1,2,3} \mathrm{M}$ Kool, ${ }^{1} \mathrm{~L}$ Kester, ${ }^{1} \mathrm{~J}$ van der Lugt. 'Princess Máxima Center, Utrecht, Netherlands; ${ }^{2}$ Hopp Children's Cancer Center (KiTZ), Heidelberg, Germany; ${ }^{3}$ Division of Pediatric Neuro-oncology, German Cancer Research Center (DKFZ) and German Cancer Consortium (DKTK), Heidelberg, Germany

\subsection{6/jitc-2021-ITOC8.19}

Background Ependymoma is the third most common brain tumor in children. At the moment, surgery and radiotherapy are the only effective treatments that can be offered, and despite this, a significant part of the patients relapse with no therapeutic salvage options. Therefore, new treatment modalities are needed. To develop immunotherapies for these children, knowledge of the tumor microenvironment is crucial. The current study aims to unravel the tumor immune microenvironment (TIME) of pediatric posterior fossa A (PFA) ependymomas.
Materials and Methods We used bulk RNA sequencing data of 22 pediatric ependymomas. We defined two groups, hereafter called PFA immune+ $(\mathrm{PFAI}+)$ and PFAI-, based on the RNA expression levels of the NanoString panel of Human PanCancer Immune Profiling genes. We performed gene set enrichment analysis and deconvoluted the bulk RNA samples with ependymoma-specific single-cell RNA sequencing datasets. To validate our findings on a protein level, we applied immunohistochemistry with antibodies recognizing tumorinfiltrating lymphocytes, tumor-associated macrophages and microglia.

Results Unsupervised hierarchical clustering of RNA expression of immune-related genes revealed two distinct PFA groups. Differential gene expression analysis showed that PFAI+ have a significantly higher expression of genes associated with immune functions, such as CD3E, CCR2, GZMA, CXCL9 and TRBC2. Accordingly, gene set enrichment analysis demonstrated that several immune pathways, including T-cell signalling, interferon-gamma response and $\mathrm{TNF} \alpha$ signalling are enriched in PFAI+ ependymomas. RNA expression of immune checkpoints was also higher in PFAI + tumors, indicating that these tumors might be more responsive to combinational therapies including immune checkpoint inhibitors. While immunohistochemistry showed low amounts of infiltrating $\mathrm{CD} 3+, \mathrm{CD} 8+$ and $\mathrm{CD} 20+$ cells, high numbers of CD163+ and HLA-DRA+ cells were detected. These cells were mainly located in regions of tumor necrosis. Increased amounts of CD4+ and CD $8+$ lymphocytes were present in PFAI+ tumors compared to PFAI- tumors. Deconvolution of the bulk RNA samples based on single-cell RNA sequencing data revealed an enrichment of myeloid cell populations, especially microglia and macrophages. Furthermore, PFAI+ tumors were found to contain significantly higher relative proportions of T-cells compared to PFAI- tumors (median of $3.76 \%$ for PFAI+ compared to $0.03 \%$ for PFAI-).

Conclusions We suggest that pediatric posterior fossa A ependymomas can be divided into two groups based on the expression of immune-related genes, in which PFAI+ ependymomas are characterized by higher RNA expression levels of these genes and greater amounts of tumor-infiltrating immune cells. Several techniques showed an enrichment of T-lymphocytes in the PFAI+ ependymomas relative to the PFAIependymomas.

Disclosure Information J. Lammers: None. F. Calkoen: None. M. Kranendonk: None. A. Federico: None. M. Kool: None. L. Kester: None. J. van der Lugt: None.

\section{P02.08 THE ROLE OF FOXP3+ REGULATORY T CELLS AND IDO+ IMMUNE AND TUMOR CELLS IN MALIGNANT MELANOMA - AN IMMUNOHISTOCHEMICAL STUDY}

${ }^{1} \mathrm{~S}$ Salmi ${ }^{*},{ }^{1} \mathrm{~A}$ Lin, ${ }^{1} \mathrm{~B}$ Hirschovits-Gerz, ${ }^{1} \mathrm{M}$ Valkonen, ${ }^{1} \mathrm{~N}$ Aaltonen, ${ }^{2,3} \mathrm{R}$ Sironen, ${ }^{4} \mathrm{H}$ Siiskonen, ${ }^{1} \mathrm{~S}$ Pasonen-Seppänen. 'Institute of Biomedicine, University of Eastern Finland, Kuopio, Finland; '2Institute of Clinical Medicine/Clinical Pathology, University of Eastern Finland, Kuopio, Finland; ${ }^{3}$ Department of Clinical Pathology, Kuopio University Hospital, Kuopio, Finland; ${ }^{4}$ Department of Dermatology, Kuopio University Hospital and University of Eastern Finland, Kuopio, Finland

10.1136/jitc-2021-ITOC8.20

Background Although Malignant Cutaneous Melanoma (CM) is a highly immunogenic cancer, it can evade the immune 